\title{
Taxoid Therapy of Breast Cancer
}

\author{
J. Crown \\ St. Vincent's University Hospital, Dublin, Ireland
}

The 1960s and early 1990s were a period of great hope for researchers who were investigating the field of systemic treatment for breast cancer. Early generation anti-metabolite/alkylatorbased combination chemotherapy regimens were demonstrated to produce high rates of objective response, with substantial palliative benefits, for patients with metastatic disease. These responses were, however, generally partial and virtually always temporary.

Throughout the 1980s and 1990s, it was generally recognised that doxorubicin was the most active agent available for the treatment of breast cancer. Between the mid 1970s, when doxorubicin was introduced into clinical practice, and the early 1990s, no further wholly new, highly active chemotherapy drugs emerged for the treatment of this disease. The 1990s have by way of contrast, been a veritable golden age in this regard, with an entirely new class of agents, the taxoids, and several active new members of existing drug classes, (e.g the vinca alkaloid vinorelbine and the antimetabolite gemcitabine) being introduced.

The taxoids, docetaxel (Taxol) and paclitaxel (Taxotere) have emerged as highly active agents and have undergone unprecedentedly rapid development. During its phase-I evaluation, the use of paclitaxel was complicated by a high incidence of hypersensitivity reactions, but the introduction of routine pre-medication resulted in a dramatic reduction in this side-effect and facilitated widespread phase-II trials. In early studies using a 24-hour infusion schedule, paclitaxel was reported to produce an approximately $50 \%$ rate of objective response in patients with untreated or minimally pre-treated metastatic breast cancer. The drug appears to be less active when administered in a 3-hour schedule. Docetaxel, which was administered in a 60-min schedule, produced a similar level of activity, but in early studies was associated with a troubling fluid retention syndrome. The use of a steroid pre-medication regimen effectively eliminated severe fluid-retention as a limiting toxicity. Both drugs have now been compared prospectively to doxorubicin in random assignment trials. In the International 303 Study, docetaxel was compared to an intensive doxorubicin program in patients whose cancer had progressed following prior alkylating agent-based therapy (adjuvant and/or metastatic). Docetaxel produced a highly statistically significantly superior response rate, and a statistically significantly superior time to treatment failure. The toxicity spectra of these drugs were very different, and generally favoured docetaxel. Doxorubicin produced a $9 \%$ incidence of clinical cardiac failure (including a $2 \%$ death rate), while there were no clinically significant decreases in ejection fraction with docetaxel treatment. Doxorubicin produced significantly more frequent and more severe thrombocytopoenia, and significantly more frequent febrile neutropenia and anaemia. The non-myeloid toxicities of these drugs were different, with doxorubicin producing significantly more frequent nausea, vomiting and stomatitis, while docetaxel produced more frequent diarrhoea and neurotoxicity. In the study of Sledge et al, paclitaxel administered in a 24-hour schedule was not statistically significantly different from doxorubicin in terms of response or survival. In the study of Paridaens et al, a 3-hour administration of paclitaxel was significantly inferior to doxorubicin in response and in progression-free survival. While firm conclusions regarding the relative merits of these drugs can only come from direct head to head comparisons (now underway), it appears that docetaxel is now the most active drug which is available for the treatment of metastatic breast cancer.

Single agent docetaxel has also been prospectively compared to three combination regimens as salvage therapy for patients whose cancer had progressed following anthracycline-based therapy. In the study of Nabholtz and colleagues, docetaxel was superior to the combination of mitomycin and vinblastine in terms of response, disease-free and overall survival. It was also compared to the regimen of intermediate-dose methotrexate and 5-fluorouracil. In this study, in which cross-over was allowed at the time of progression (rendering overall survival comparisons difficult), docetaxel was associated with a statistically significantly superior response and time to progression. Single agent docetaxel was also demonstrated to be equivalent to a two-drug combination consisting of long-term infusional 5-fluorouracil and vinorelbine (both active single agents in anthracycline failures).

Taxoid-containing combination chemotherapy regimens have been the subject of particular interest. Using a paclitaxel/ doxorubicin combination, Gianni and colleagues reported more than $90 \%$ objective responses, including $41 \%$ complete remissions in patients with chemotherapy-naive metastatic breast cancer, with a substantial incidence of clinical cardiac

\begin{tabular}{ll}
\hline KARGER & @ 1999 S. Karger GmbH, Freiburg \\
$\begin{array}{l}\text { Fax +497614520714 } \\
\begin{array}{l}\text { E-mail kargergmbh@aol.com } \\
\text { www.karger.com }\end{array}\end{array}$ & Accessible online at: \\
http://BioMedNet.com/karger
\end{tabular}

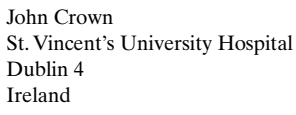


toxicity. In other studies in which more traditional eligibility criteria were used (i.e. prior adjuvant allowed), the response rates were closer to $50 \%$ (table 1 ). The combination of docetaxel and doxorubicin has also been studied. Dieras et al. and Nabholtz et al. (whose study also included cyclophosphamide) reported response rates which were in excess of $70 \%$ in metastatic breast cancer patients, approximately $40 \%$ of whom had had prior adjuvant chemotherapy. Nabholtz has also recently reported that the combination of docetaxel and doxorubicin produced a statistically significantly higher rate of objective response than did the combination of doxorubicin and cyclophosphamide.

The chemotherapy of metastatic breast cancer remains an essentially palliative undertaking, but might the same regimens which produce responses in metastatic disease have a greater impact if used in patients with earlier stage cancer? Drawing from the experience of the classical pre-clinical studies of Skipper and Schabel, it was anticipated that such 'adjuvant' therapy might have a substantial curative impact in the postoperative treatment of patients with node-positive disease. While adjuvant combination chemotherapy has been demonstrated to prolong disease-free and overall survival in this setting, the impact has been less than might have been hoped for. Thus, the study of the taxoids as components of adjuvant regimens was an obvious next step, and it is interesting to note that all of the large North American adjuvant studies which are now being planned include taxoids. Already, preliminary results from the Intergroup Study in the USA suggest that a regimen in which patients with node-positive disease 'crossover' to paclitaxel following doxorubicin-cyclophosphamide (AC) is superior to AC alone as adjuvant therapy. The high activity, and incomplete cross-resistance between docetaxel and the anthracyclines makes docetaxel/doxorubicin combinations particularly appealing, and several such studies are underway. In a pilot evaluation, investigators in Brussels and Dublin demonstrated the feasibility of a candidate crossover regimen in which 3 cycles of doxorubicin $\left(75 \mathrm{mg} / \mathrm{m}^{2}\right)$ were followed by 3 cycles of docetaxel $\left(100 \mathrm{mg} / \mathrm{m}^{2}\right)$, both at 3 -week intervals, with a further cross-over to classic day 1 and day $8 \mathrm{CMF}$ schedule (all i.v.). This sequential regimen is being compared to combination AT followed by $\mathrm{CMF}$, and to 2 non-taxoid-containing anthracycline-based regimens in the international BIG study.

In recent years there has been considerable interest in the use of high-dose chemotherapy with haematopoietic autograft support as a treatment for metastatic and for node-positive breast cancer. Large-scale confirmatory random assignment trials are underway in an attempt to see if the promising results of early single arm historically-controlled studies can be validated. It is increasingly obvious that such studies must contain optimised control arms if the results are to be interpretable. In the European Breast Cancer Dose Intensity Study, patients
Tab. 1. Effects of Taxotere and Doxorubicin, all values in percent $(\mathrm{p}=0.008)$

\begin{tabular}{lcc}
\hline & $\begin{array}{l}\text { Taxotere } \\
(\mathrm{n}=161)\end{array}$ & $\begin{array}{l}\text { Doxorubicin } \\
(\mathrm{n}=165)\end{array}$ \\
\hline Complete remission & 7 & 4 \\
Partial remission & 41 & 29 \\
Response rate & 48 & 33 \\
Stable disease & 34 & 38 \\
Progressing disease & 12 & 22 \\
No effect & 6 & 7 \\
\hline
\end{tabular}

with chemotherapy naive (prior adjuvant is allowed) metastatic breast cancer receive either 4 cycles of AT followed by CMF; or 3 cycles of AT followed by tandem applications of high-dose chemotherapy. In a pilot evaluation, it was demonstrated that AT with lenograstim support mobilizes haematopoietic progenitors into the peripheral blood, and that the high-dose regimen was feasible, tolerable and active.

One of the advantages which investigators working with docetaxel have enjoyed has been the early identification of a standard dose and schedule for this drug, namely $100 \mathrm{mg} / \mathrm{m}^{2}$ given every 3 weeks via a 60-min intravenous infusion. However, it is possible that non-classical scheduling of taxoids might result in favourable alterations in the therapeutic ratio. For instance, weekly lower-dose paclitaxel has been demonstrated to produce high rates of response in breast and ovarian cancer, with little myelosuppression, but with dose-limiting neurotoxicity. Weekly lower-dose docetaxel has also been studied, and again has produced promising activity in metastatic breast cancer. In the case of this drug, fatigue, not neurotoxicity has been dose-limiting.

Recently, the first reports of activity for a 'tailored' monoclonal antibody in the treatment of breast cancer have been reported. Herceptin, which reacts with the protein product of the her2 -neu oncogene has produced approximately $15 \%$ objective responses in pre-treated, her-2-neu over-expressing, patients with metastatic disease. Interestingly, an additive effect was seen when this drug was combined with paclitaxel. Any possible synergistic or additive interactions between Herceptin and docetaxel would obviously be of great interest, and these are being addressed in ongoing and planned studies.

One of the more heartening aspects of the taxoid story has been the rapid pace of clinical development which these products have undergone. It is very impressive that in the first decade following the determination of safe schedules for their administration, that their use in combination therapy in metastatic disease is already well established, and that their study in the adjuvant setting is well underway, with promising preliminary results. 